\title{
NEUTRON TUBE DESIGN STUDY FOR BORON NEUTRON CAPTURE THERAPY APPLICATION"
}

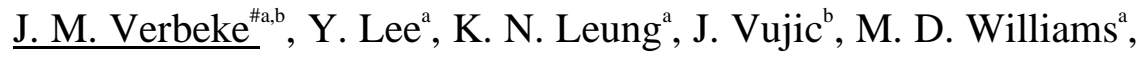 \\ L. K. Wu and N. Zahir ${ }^{\mathrm{a}, \mathrm{b}}$ \\ ${ }^{a}$ Lawrence Berkeley National Laboratory \\ ${ }^{\mathrm{b}}$ Nuclear Engineering Department, University of California, Berkeley \\ Berkeley, CA 94720 \\ USA
}

\begin{abstract}
Radio-frequency (RF) driven ion sources are being developed in Lawrence Berkeley National Laboratory (LBNL) for sealed-accelerator-tube neutron generator application. By using a 5-cm-diameter RF-driven multicusp source $\mathrm{H}^{+}$yields over $95 \%$ have been achieved. These experimental findings will enable one to develop compact neutron generators based on the D-D or $\mathrm{D}-\mathrm{T}$ fusion reactions. In this new neutron generator, the ion source, the accelerator and the target are all housed in a sealed metal container without external pumping. Recent moderator design simulation studies have shown that $14 \mathrm{MeV}$ neutrons could be moderated to therapeutically useful energy ranges for boron neutron capture therapy (BNCT). The dose near the center of the brain with optimized moderators is about $65 \%$ higher than the dose obtained from a typical neutron spectrum produced by the Brookhaven Medical Research Reactor (BMRR), and is comparable to the dose obtained by other accelerator-based neutron sources. With a $120 \mathrm{keV}$ and 1 A deuteron beam, a treatment time of $\sim 35$ minutes is estimated for BNCT.
\end{abstract}

\section{INTRODUCTION}

The RF-driven multicusp ion source developed at Lawrence Berkeley National Laboratory has found numerous applications ranging from neutral beam injection systems for fusion reactors to particle accelerators, proton therapy machines and ion implantation systems [1]. Such sources are simple to operate, have long lifetimes, high gas efficiencies and provide high density plasmas with high monatomic species yields. These characteristics make the RF-driven ion source a viable candidate for the next generation of compact, high-output, sealed-tube neutron generators, utilizing the fusion of deuterium and tritium, or deuterium and deuterium.

\footnotetext{
This work is supported by Sandia National Laboratory and the US Department of Energy under contract No. DE-AC03-76F00098.

${ }^{\text {"}}$ Email: jmverbeke@1bl.gov
}

Recently, LBNL has developed a compact, sealedaccelerator-tube neutron generator capable of producing a neutron flux in the range of $10^{9}$ to $10^{10} \mathrm{D}$-T neutrons per second [2-3]. The ion source, a miniaturized variation of earlier RF-driven multicusp ion sources, is designed to fit within a 5-cm-diameter borehole. Typical operating parameters include repetition rates up to 100 pps, with pulse widths between 10 and $80 \mu$ s (limited only by the available RF power supply) and source pressure as low as 5 mTorr. In this configuration, peak extractable hydrogen current densities exceeding 1 $\mathrm{A} / \mathrm{cm}^{2}$ with $\mathrm{H}^{+}$ion yields over $94 \%$ have been achieved. From this output, a D-T neutron yield of $10^{9}$ neutrons per second can be projected. Simple scaling of the ion source and extraction aperture size could bring the neutron output even higher.

These experimental findings together with recent ion source testing and moderator design [4] will enable one to develop compact $14 \mathrm{MeV}$ neutron generators based on the D-T fusion reaction. In this new system, the ion source, the accelerator and the target are all housed in a sealed metal container without pumping. With a $120 \mathrm{keV}$ and $1 \mathrm{~A}$ average $\mathrm{D}^{+}$beam current, it is estimated that a treatment time of $\sim 35$ minutes is needed for boron neutron capture therapy $\left(10^{14}\right.$ neutrons/sec $)$. This article describes the design and characteristics of the new neutron generator.

\section{NEUTRON TUBE DESIGN}

In order to achieve a neutron yield of $10^{14}$ neutrons/sec, a large multicusp source together with a multi-aperture extraction system to produce an ion beam current of $1 \mathrm{~A}$, accelerated to $120 \mathrm{kV}$, and impinging on a well-cooled target is required.

The main components of the sealed D-T neutron tube are the ion source, the $120 \mathrm{kV}$ accelerator column, the water-cooled target and the vacuum system. Figure 1 shows a schematic diagram of the sealed D-T neutron generator. It is a scale up version of the compact neutron tube that LBNL has developed. The characteristics of this neutron generator are as follows. 


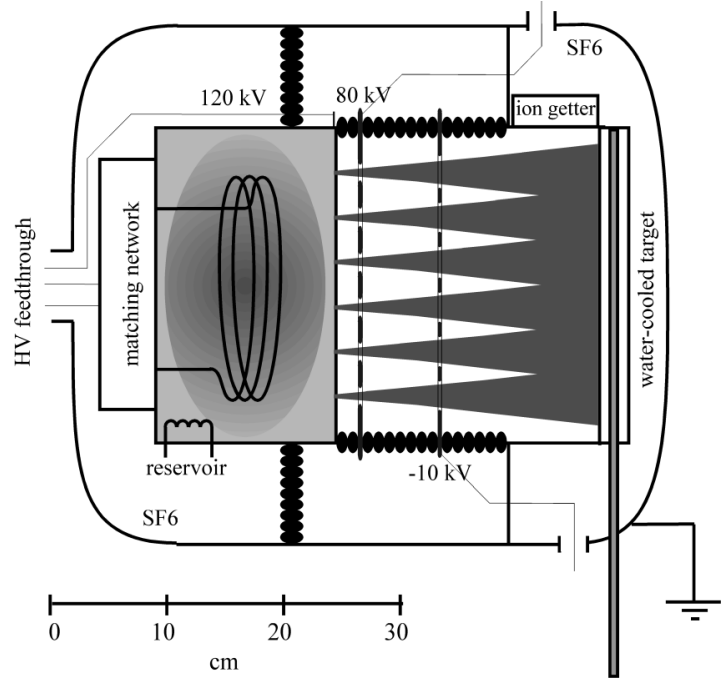

Figure 1: Schematic diagram of the sealed D-T neutron generator.

\subsection{The ion source}

The multicusp generator is a 30-cm-diameter cylindrical stainless-steel chamber surrounded with columns of samarium-cobalt magnets. The plasma is produced by RF induction discharge. In order to deliver RF power to the plasma, a coupler in the form of a multiturn induction coil is used. The RF power supply is a broad band power amplifier driven at $13.56 \mathrm{MHz}$ by a signal generator.

To maximize the neutron output at the target, it is necessary to produce high $\mathrm{D}^{+}$and $\mathrm{T}^{+}$concentrations in the extracted beam. Experiments have been carried out with a 5-cm-diameter ion source to determine the fractions of hydrogen ion species. For a hydrogen pressure of 4 mTorr, the results are plotted in Fig. 2.

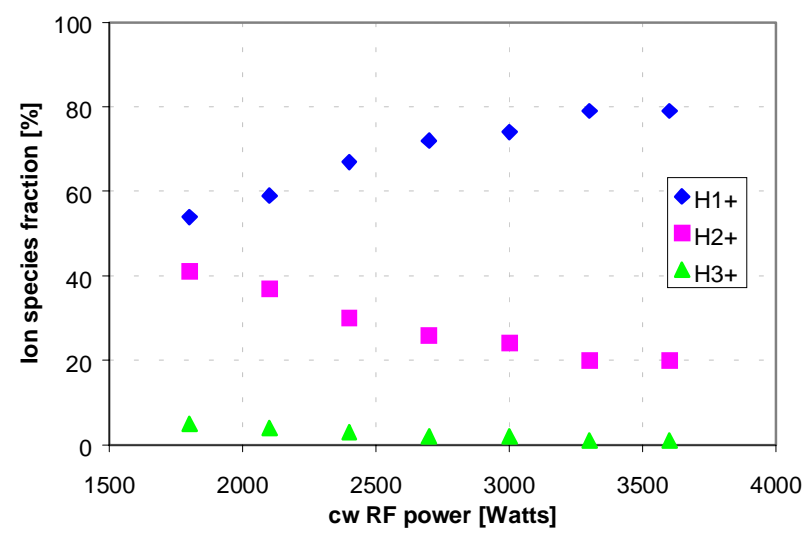

Figure 2: Hydrogen ion species fractions versus cw RF power for gas pressure of 4 mTorr, without magnetic filter.

The monatomic ion species $\mathrm{H}^{+}$can be enhanced by installing a permanent magnetic filter in the source chamber [3]. Lower RF power is then needed to sustain the plasma: $660 \mathrm{~W}$ instead of $1800 \mathrm{~W}$ without magnetic filter. For an input RF power of $1500 \mathrm{~W}$, the minimum gas pressure to sustain the plasma also decreases, from 4 mTorr down to 2 mTorr.

Fig. 3 shows that the filter equipped source produces a higher $\mathrm{H}^{+}$fraction than the source without magnetic filter.

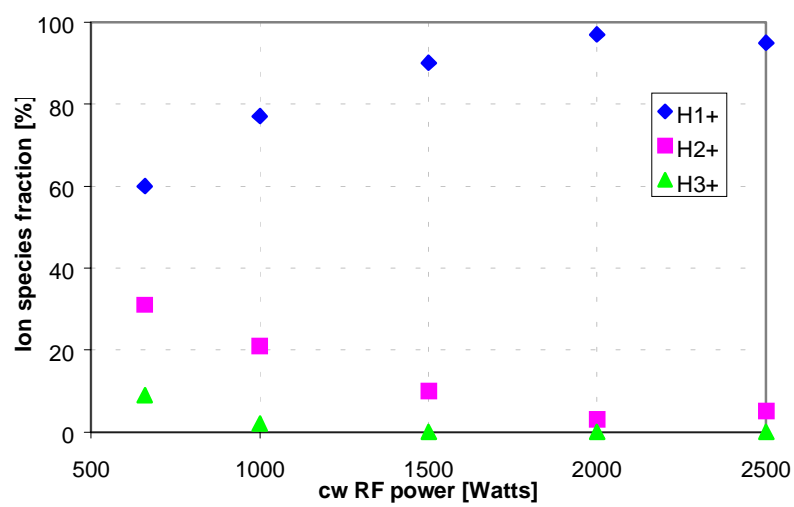

Figure 3: Hydrogen ion species fractions versus cw RF power for gas pressure of $4 \mathrm{mTorr}$, with magnetic filter.

In the best case, we obtained $97 \%$ of $\mathrm{H}^{+}$, to be compared with $79 \%$ without magnetic filter. Fig. 4 shows the hydrogen ion species for this particular case.

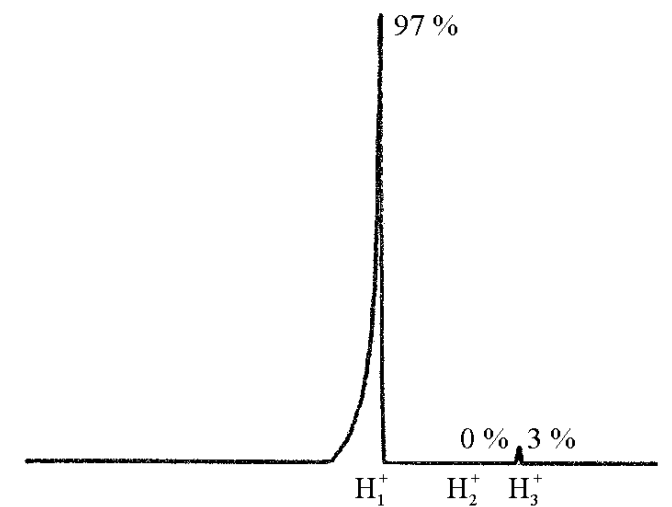

Figure 4: Hydrogen ion species for $\mathrm{cw} R F$ power= $2 \mathrm{~kW}$ and gas pressure of 4 mTorr.

Apart from an area of about $5 \mathrm{~cm}$ from the chamber wall, the plasma density is very uniform in the central region of the 30-cm-diameter source. Thus, one can form multiple beamlets with good ion optics to enhance the extractable current.

Figure 5 shows the current density increasing linearly with the RF power. The magnetic filter strength was lower for these measurements due to prior overheating of the magnets. For an input RF power of $3 \mathrm{~kW}$ and assuming $50 \%$ transparency, we can estimate a total extraction area of $\sim 60 \mathrm{~cm}^{2}$ for a current density of 33 $\mathrm{mA} / \mathrm{cm}^{2}$.

We plan to operate the source in a pulsed mode with a $10 \%$ duty factor at a current density of $150 \mathrm{~mA} / \mathrm{cm}^{2}$. This will produce an average beam current of $1 \mathrm{~A}$ on the 
target. The RF input power for the beam pulse is now higher, the operating pressure should decrease and the atomic species fraction should improve.

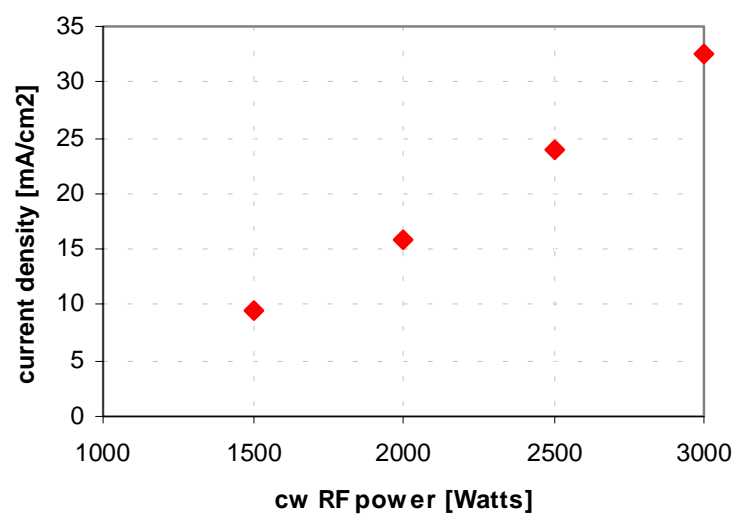

Figure 5: Current density versus cw RF power for gas pressure of 4 mTorr.

\subsection{The $120 \mathrm{kV}$ accelerator column}

The beam extraction system which closes off the other end of the ion source chamber, contains three electrodes with multiple 5-mm-diameter apertures. The shape, the separation and the voltage distribution on these electrodes are designed by using the I-GUN computation code. The beamlets expand after they exit from the last electrode and therefore the power can be spread uniformly across the target. Source and electrode damages caused by high energy backstreaming electrons can be avoided by the presence of a suppressor electrode.

\subsection{The water-cooled target}

The target is a water-cooled copper plate covered by a layer of titanium which can absorb deuterium and tritium atoms efficiently. Since the concentrations of deuterium and tritium in titanium decrease with increasing temperature, it is important to keep the target as cold as possible. With a $400 \mathrm{~cm}^{2}$ target area, the power density on the target due to the impinging $\mathrm{D}^{+}$and $\mathrm{T}^{+}$ions is $\sim 300$ $\mathrm{W} / \mathrm{cm}^{2}$ which can be easily handled by a modest water flow-rate. In order to maximize the lifetime of the target and to maintain a constant neutron output, a self-loading target will be used and the multicusp source will be operated with a $50 \%-50 \%$ mixture of deuterium and tritium.

In normal operation, the entire tube will be at an ambient pressure of $\sim 2$ mTorr. Low gas pressure is essential for reducing both charge exchange and highvoltage break-down in the accelerator column. The deuterium and tritium gas pressures are controlled by a reservoir element consisting of a tungsten wire coated with zirconium. Release of deuterium and tritium gases is achieved by circulating a current in the tungsten wire. After operation, deuterium and tritium will return to the reservoir element and target due to the decrease of their temperature. The absence of gaseous tritium in the tube between operations makes the sealed neutron tube safe. In order to control the helium pressure buildup in the tube, some getter pumps will be installed.

Since there is no weak component, the lifetime of the tube should be very long. Higher neutron output can be achieved either by increasing the extraction area or by operating the source with higher discharge power.

\section{MODERATOR DESIGN}

Epithermal neutrons of energy $10 \mathrm{keV}$ are required for BNCT applications. Using a D-T neutron source of energy $14.1 \mathrm{MeV}$, one can generate the desired neutron energy spectrum by optimization of the moderator [4].

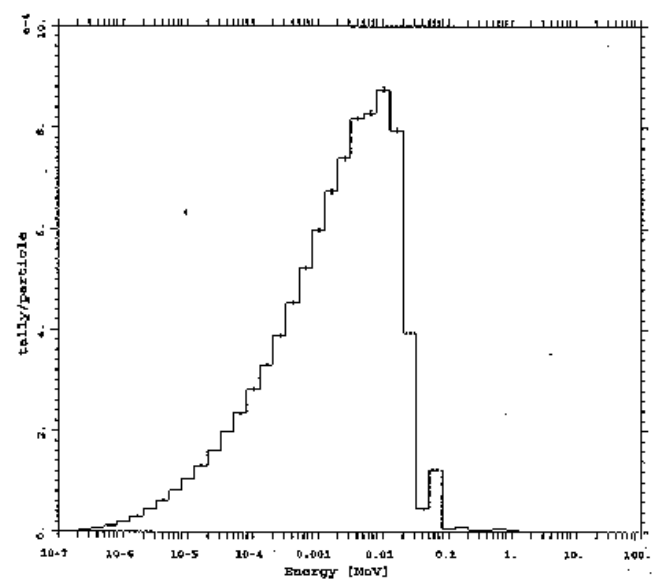

Figure 6: Neutron spectrum obtained after moderation of D-T neutrons.

The neutron spectrum obtained by simulation shown in Fig. 6 results in a dose to the center of the brain about $65 \%$ higher than the dose obtained from a typical neutron spectrum produced by the Brookhaven Medical Research Reactor (BMRR), and comparable to the dose obtained by other accelerator-based neutron sources. One can estimate a treatment time of $\sim 35$ minutes with a $10^{14}$ $\mathrm{n} / \mathrm{sec}$ neutron output.

\section{ACKNOWLEDGEMENTS}

We would like to thank members of the Plasma and Ion Source Technology Group at LBNL for all the technical assistance. This work is supported by Sandia National Laboratory and the US Department of Energy under contract No. DE-AC03-76F00098.

\section{REFERENCES}

[1] K. N. Leung, Rev. Sci. Instrum., 67, 1302, (1996).

[2] L. T. Perkins, P. R. Herz, K. N. Leung and D. Pickard, Rev. Sci. Instrum., 65, 1186, (1996).

[3] L. T. Perkins, et.al., Rev. Sci. Instrum., 67, 1057 (1996).

[4] J. M. Verbeke, J. Vujic and K. N. Leung, Proc. of the $8^{\text {th }}$ Int. Symp. on Neutron Capture Therapy for Cancer, La Jolla, CA, Sept. 1998. 